\title{
CORRIGENDUM
}

\section{A Fast Adaptive-Gain Complementary Filter Algorithm for Attitude Estimation of an Unmanned Aerial Vehicle - CORRIGENDUM}

\author{
Qing-quan Yang, Ling-ling Sun, and Longzhao Yang
}

doi.org/10.1017/S0373463318000231, Published by Cambridge University Press, 21 May 2018.

The authors of "A Fast Adaptive-Gain Complementary Filter Algorithm for Attitude Estimation of an Unmanned Aerial Vehicle" first published online on 21 May 2018 (Yang et al., 2018) have drawn attention to the fact that some of the work insufficiently acknowledges the work of Wu et al. (2016). Section 2.2 of Yang et al. (2018) builds on the work of Wu et al. (2016).

In Yang et al. (2018), the authors derive an observation model of attitude quaternions from normalised accelerometer measurements. The method of Yang et al. builds up the linear measurement model via Taylor approximations. Some significant contributions and new filter designs are shown in this paper which deal with the problem of adaptive estimation. Regarding the factorization of the direction cosine matrix, it has been systematically described in Wu et al. (2018), but this has not been noticed by Yang et al. as this paper was published after the original submission of Yang et al. (2018). Yang et al. (2018) uses different compositions of a dynamical system that achieves the same result as in Wu et al. (2016).

Yang et al. would like to apologise to $\mathrm{Wu}$ et al. and the editorial board of the Journal of Navigation for their failure to properly cite the earlier work and acknowledge the originality of Wu et al.'s work.

\section{REFERENCES}

Wu, J., Zhou, Z., Chen, J., Fourati, H. and Li, R. (2016). Fast Complementary Filter for Attitude Estimation Using Low-Cost MARG Sensors. IEEE Sensors Journal, 16(18), 6997-7007.

Wu, J., Zhou, Z., Gao, Bin, Rui Li, and Hassen Fourati. (2018). Fast Linear Attitude Estimator Using Vector Observations. IEEE Transactions on Automation Science and Engineering, 15(1), 307-319.

Yang, Q., Sun, L. and Yang, L. (2018). A fast adaptive-gain complementary filter algorithm for attitude estimation of an unmanned aerial vehicle. The Journal of Navigation, Published by Cambridge University Press, 21 May 2018. doi.org/10.1017/S0373463318000231. 